\title{
Facial emotion recognising memristive threshold logic system
}

\author{
Dayana Mathew, Dinesh Sasi Kumar, Alex Pappachen James
}

- School of Engineering

\begin{abstract}
The edge features in facial images change with changes in images, and are useful for automatically recognising the facial expressions. We present an edge feature extraction method using neuronal threshold logic models to automatically recognise the facial expressions. The system is simulated at digital system level consisting of reading an image followed up with edge extraction system that can be implemented with hybrid CMOSmemristive digital circuits. The results indicate robust edges of the facial features and its useful in development of real-time emotion recognizing digital chip.
\end{abstract}

Original language

Title of host publication

Publisher

Pages

Number of pages

ISBN (Electronic)

State

Event
English

2015 IEEE Recent Advances in Intelligent Computational Systems, RAICS 2015

$\underline{\text { Institute of Electrical and Electronics Engineers Inc. }}$

$39-44$

6

9781467366700

Published - Jun 92016

2015 IEEE Recent Advances in Intelligent Computational Systems, RAICS 2015 - Trivandrum, Kerala, India

Mathew, D., Kumar, D. S., \& James, A. P. (2016). Facial emotion recognising memristive threshold logic system. In 2015 IEEE Recent Advances in Intelligent Computational Systems, RAICS 2015. (pp. 39-44). [7488385] Institute of Electrical and Electronics Engineers Inc.. DOI: 10.1109/RAICS.2015.7488385 